\title{
Zur Kenntnis des sogenannten Molybdändichlorids.
}

Von

\author{
Arthur Rosenherm und Franz Kohn. \\ (VIII. Nitteilung: Über die Halogenverbindungen des Molybdäns \\ und Wolframs. $)^{1}$
}

BLOMSTRAND ${ }^{2}$ beschreibt in seinen ausführlichen Untersuchungen über die Halogenverbindungen des Molybdäns ein Chlorid bzw. Bromid, das auf jedes Atom Molybdän zwei Atome Halogen im Molekül enthält. Das eigentümliche Verhalten dieser Stoffe, die durch Erhitzen der entsprechenden Trihalogenide im Kohlensäurestrom und dabei eintretender Zerlegung derselben in die vorliegenden Verbindungen und Tetrahalogenide dargestellt werden, ihre grofse Beständigkeit gegen Oxydationsmittel wie Salpetersäure und Königswasser, die anormalen Reaktionen eines Teiles des in ihnen enthaltenen Halogens veranlassen ihn, die Bruttoformel zu verdreifachen und sie mit der Formel $\left[\mathrm{Mo}_{3} \mathrm{Cl}_{4}\right] \mathrm{Cl}_{2}$ bzw. $\left[\mathrm{Mo}_{3} \mathrm{Br}_{4}\right] \mathrm{Br}_{2}$ als Chlorid bzw. Bromid komplexer zweiwertiger Kationen aufzufassen. BLoMSTRand stellte zahlreiche Salze, Doppelsalze und Hydrate dieser Verbindungen her, die diese Annabme zwar bestätigten, jedoch zum Teil analytisch nicht einwandsfrei untersucht sind, wie weiter unten nachgewiesen werden wird. Ein Schüler Blomstrands A. AtterBERG $^{3}$ beschrieb dann bald darauf in einer nicht mehr zugänglichen Dissertation ${ }^{4}$ zahlreiche Salze des bromhaltigen Kations. Wenig später untersuchten dann P. Liechti und B. KEMPE ${ }^{5}$ die wasserfreie Chlorverbindung und ein daraus entstehendes Hydrat und bekamen für letzteres Werte, die von den Resultaten Buomstrands etwas abweichen. Endlich haben sich W. Muthmann und W. NaGeL ${ }^{6}$ mit diesen Verbindungen beschäftigt und besonders durch ebullioskopische

1 VII. Mitteilung: Z. anorg. Chem. 65, 166.

2 Journ. prakt. Ohem. 71, 449; 77, 88; 82, 433.

3 Jahresberichte 1872, 260.

- Upsala.

${ }^{5}$ Lieb. Ann. 169 (1873), 354.

${ }^{6}$ Ber. deutsch. chem. Ges. 31 (1898), 2009.

Z. anorg. Chem. Bd. 66 . 
Molekulargewichtsbestimmungen des wasserfreien Chlorids in absolutem Alkohol die trimolekulare Formel BuoMstrands experimentell bestätigt.

Zur Darstellung des wasserfreien Chlorids wurde bei den vorliegenden Versuchen metallisches Molybdän verwandt, das nach dem aluminothermischen Verfahren gewonnen wurde. ${ }^{1}$ Es gelang trotz zablreicher Versuche bisher nicht das schon von Blomstrand angegebene und von den anderen Bearbeitern dieses Gegenstandes angewandte Darstellungsverfahren für das Cblorid, das besonders in bezug auf die Ausbeute sehr wenig befriedigt, wesentlich zu verbessern. Zur Erhitzung der Chloride wurde ein elektrischer Drahtwiderstandsofen verwendet, der eine gleichmälsigere Temperaturregulierung gestattete als die Gasverbrennungsöfen.

Das wasserfreie gelbe Molybdändichlorid ist aufserordentlich schwer rein zu erhalten; es enthält meist andere Molybdänchloride beigemengt, die nur durch Behandlung mit mälsig verdünnter Salpetersäure oder auf mechanischem Wege getrennt werden können. Es löst sich in konzentrierter Salzsäure leicht auf: mit tiefgelber Farbe, wenn es rein ist, mit blaugrüner wenn andere Chloride beigemengt sind, und aus dieser Lösung erhält man beim Einengen auf dem Wasserbade tiefgelbe Krystallnadeln, die BLOMstrand allerdings nur auf Grund einer Molybdän- und einer Trockenrückstandsbestimmung als ein Hydrat $\mathrm{MO}_{3} \mathrm{Cl}_{6} .6 \mathrm{H}_{2} \mathrm{O}$ ansprach.

Von dieser Verbindung wurden gröfsere Mengen dargestellt und durch Umkrystallisieren aus Chlorwasserstoffsäure 1.12 gereinigt.

Die sorgfältigen Analysen dieser Verbindung führten nun zu Resultaten, die mit der von Blomstrand angenommenen Formel nicht in Einklang zu bringen waren. Sie wurden auf verschiedenen Wegen gewonnen. Die abgewogene Substanz wurde in überschüssigem Ammoniak gelöst, das Molybdän durch Zusatz von etwas Ammoniumpersulfat oxydiert, die Lösung mit Schwefelammonium digeriert und durch Ansäuern mit verdünnter Schwefelsäure Molybdänsulfid gefällt, das als Molybdäntrioxyd zur Wägung gebracht wurde. Im Filtrate wurde das Chlor bestimmt. Bei anderen Bestimmungen wurde die Substanz im Wasserstoffstrome erhitzt, der entweichende Chlorwasserstoff in Ammoniak absorbiert und als Silberchlorid bestimmt und das zurückbleibende

' A. Rosenhem und H. Braun, Z. anorg. Chem. 46 (1905), 311. 
metallische Molybdän gewogen. Die zahlreichen Bestimmungen ergaben übereinstimmend die Formel:

$$
\begin{aligned}
& \mathrm{MO}_{3} \mathrm{Cl}_{6} \cdot \mathrm{HCl} \cdot\left(\mathrm{H}_{2} \mathrm{O}\right)_{4} \text {. } \\
& \text { Mo: } \quad \begin{array}{llllll}
47.80 & 47.69 & 47.70 & 47.76 & 47.53 \%
\end{array} \\
& \mathrm{Cl}: \quad \begin{array}{lllllll}
40.54 & 40.81 & 40.79 & 40.27 & 40.60 & 40.03 & 39.98 \%
\end{array} \\
& \text { Berechnet: Gefunden im Mittel: } \\
& \begin{array}{lll}
\mathrm{Mo} & 47.28 & 47.69 \% \\
\mathrm{Cl} & 40.73 & 40.43 \\
\mathrm{H}_{2} \mathrm{O} & 11.99 & 11.88
\end{array}
\end{aligned}
$$

Nach diesem Resultat unterscheidet sich die Formel der Substanz von der Annahme Blomstrands durch den Ersatz zweier Mol. $\mathrm{H}_{2} \mathrm{O}$ durch $1 \mathrm{Mol}$. $\mathrm{HCl}$, was infolge der annähernden Gleichheit der Molekulargewichte $\left[\left(\mathrm{H}_{2} \mathrm{O}\right)_{2}=36 ; \mathrm{HCl}=36.5\right]$ bei den von BLoMSTRAND ausgeführten Bestimmungen des Molybdängehaltes und Trockenrückstandes nicht zutage treten konnte.

Dafs die Verbindung tatsächlich Chlorwasserstoff gebunden enthält, geht daraus hervor, dafs sie im geschlossenen Gefälse in einer Chlorwasserstoffatmosphäre absolut haltbar ist, an der Luft unter Annahme einer grünlichen Färbung Salzsäure schnell abgibt, dann aber beim Überleiten von gasförmiger Salzsäure die ursprüngliche tiefgelbe Farbe wieder annimmt. Liechis und Kempe hatten schon beobachtet, ${ }^{1}$ dafs „den Krystallen etwas Salzsäure hartnäckig anhaftet" und hatten dadurch, dafs sie diese Beobachtung nicht genügend berücksichtigten, schwankende Analysenwerte erbalten, welche die sichere Aufstellung einer Formel nicht zuliefsen.

Um zur weiteren Aufklärung dieser komplizierten Bruttoformel die Wertigkeit des Molybdäns in dieser Verbindung zu ermitteln, wurden gewogene Mengen der Substanz mit ammoniakalischer Silberlösung digeriert, wobei unter Abscheidung äquivalenter Mengen metallischen Silbers das Molybdän zu sechswertigem Molybdän oxydiert wird.

$0.5123 \mathrm{~g}$ Substanz ergaben auf diese Weise $1.0598 \mathrm{~g} \mathrm{Ag}$

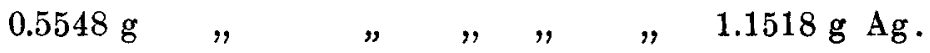

Aus diesen Bestimmungen folgt, dafs

I. $1 \mathrm{Mol} . \mathrm{Mo}_{3} \mathrm{Cl}_{6} \mathrm{HCl} .4 \mathrm{H}_{2} \mathrm{O}$ (Molgew. $=609.5$ ) 11.7 Atome $\mathrm{Ag}$ II. $1, \mathrm{Mo}_{3} \mathrm{Cl}_{6} \mathrm{HCl}_{4} \mathrm{H}_{2} \mathrm{O} \quad 11.9 \quad " \mathrm{Ag}$ reduzieren. Die drei im Mol. der Verbindung enthaltenen Mo- 
lybdänatome nehmen beim Übergang in sechswertiges Molybdän 12 Valenzen auf und mithin liegt, der bisher experimentell noch nicht bewiesenen Annahme Blomstrands entsprechend, eine Verbindung des zweiwertigen Molybdäns vor.

Wurden weiterhin gewogene Mengen der Substanz im Chlorwasserstoffstrome, um eine Zersetzung in Oxychloride möglichst zu vermeiden, erwärmt, so trat schon bei $100^{\circ}$ Gewichtskonstanz ein, und weiteres Erhitzen bis $130^{\circ}$ brachte keine weitere Veränderung hervor, als dafs die Substanz sich dabei etwas dunkler färbte, während sie beim Abkühlen aber die hellgelbe Farbe wieder annahm. Es zeigte sich, dals das angelagerte Mol. Chlorwasserstoffsäure und $3 \mathrm{Mol}$. Wasser abgespalten wurden, während $1 \mathrm{Mol}$. Wasser fest gebunden blieb.

'l'rockenverlust berechnet für $\mathrm{HCl}+3 \mathrm{H}_{2} \mathrm{O}: \quad 14.81 \%$

gefunden bei $100-130^{\circ}$ : $14.84 \quad 14.71 \quad 14.97 \%$

Die Analysen des Trockenrückstandes bestätigten diese Ergebnisse, denn derselbe entsprach in seiner Zusammensetzung der Formel

\begin{tabular}{lccc} 
& \multicolumn{3}{c}{$\mathrm{Mo}_{3} \mathrm{Cl}_{6} \cdot \mathrm{H}_{2} \mathrm{O}}$. \\
& Berechnet: & \multicolumn{3}{c}{ Gefunden: } \\
$\mathrm{Mo}$ & 55.52 & 56.44 & 55.88 \\
Cl & 41.02 & 40.90 & $41.10 \quad 41.30 \%$ \\
$\mathrm{H}_{2} \mathrm{O}$ & 3.46 & & $2.74 \%$
\end{tabular}

Wird die Verbindung längere Zeit über $130^{\circ}$ anf ca. $200^{\circ}$ erhitzt, so tritt teilweise Zersetzung ein, sie verliert auch das letzte Mol. Wasser. (Trockenverlust: $18.84 \%$; berechnet für $\mathrm{HCl}+4 \mathrm{H}_{2} \mathrm{O}$ : $17.81 \%$.) Beim Glühen wird die Substanz vollständig zersetzt.

Nach diesen Ergebnissen mürste man der Verbindung die Konstitutionsformel

$$
\left[\mathrm{Mo}_{3} \mathrm{Cl}_{6} \cdot \mathrm{H}_{2} \mathrm{O}\right] \cdot \mathrm{HCl} \cdot 3 \mathrm{H}_{2} \mathrm{O}
$$

zuerteilen, wenn nicht Blomstrand nachgewiesen hätte, dals der hier ein "Kern" enthaltene Bestandteil $\mathrm{Mo}_{3} \mathrm{Cl}_{6}$ seinerseits noch zwei ionisierbare Chloratome enthielt. Tatsächlich bestätigte sich diese Beobachtung auch für die vorliegende Verbindung, wenn man wie Blomstrand verfuhr. Die Substanz wurde in Kalilauge gelöst, die klare Lösung mit Essigsäure versetzt und der ausfallende gelbweifse Niederschlag abfiltriert. Eine Chlorbestimmung im Filtrat zeigte, dafs von den sieben Chloratomen der Verbindung $\mathrm{Mo}_{3} \mathrm{Cl}_{6}$. 
$\mathrm{HCl} .4 \mathrm{H}_{2} \mathrm{O}$ tatsächlich drei gefällt waren und mithin der gelbweifse Niederschlag die Formel $\mathrm{Mo}_{3} \mathrm{Cl}_{4}(\mathrm{OH})_{2}$ haben mufste.

Berechnet $\mathrm{Cl}_{3}$ aus $\mathrm{Mo}_{3} \mathrm{Cl}_{6} \mathrm{HCl} .4 \mathrm{H}_{2} \mathrm{O}: \quad 17.46 \%$.

$$
\text { Gefunden: } 17.96 \% \text {. }
$$

Diese Methode zur Bestimmung der ionisierbaren Chloratome ist jedoch etwas gewaltsam. Die Verbindung $\mathrm{Mo}_{3} \mathrm{CJ}_{6} \cdot \mathrm{HCl} .4 \mathrm{H}_{2} \mathrm{O}$ löst sich mit gelber Farbe klar in kaltem Wasser; nach wenigen Minuten jedoch scheidet sich aus dieser Lösung ein feinpulveriger gelber Niederschlag $a b$, der allerdings erst durch längeres Erwärmen der Lösung vollständig zur Abscheidung gebracht wird. Im Filtrate einer solchen Fällung, die durch mehrstündiges Erhitzen herbeigeführt war, ergab eine Chlorbestimmung eine wesentlich geringere Menge dissoziierten Chlors.

$$
\begin{aligned}
& \text { Berechnet } \mathrm{HCl} \text { aus } \mathrm{Mo}_{3} \mathrm{Cl}_{6} \cdot \mathrm{HCl} .4 \mathrm{H}_{2} \mathrm{O}: 5.82 \% \text {. } \\
& \text { Gefunden: } 9.01 \% \text {. }
\end{aligned}
$$

Hiernach erscheint es selrr wahrscheinlich, dafs ursprünglich durch Wasser nur das Mol. Chlorwasserstoff abgespalten wird und dals folglich die obige Konstitutionsformel den experimentellen Beobachtungen entspricht. Über die Konstitution des Kerns: $\left[\mathrm{Mo}_{3} \mathrm{Cl}_{6}\right.$. $\left.\mathrm{H}_{2} \mathrm{O}\right]$ lälst sich vorläufig nichts sagen.

Blomstrand hat eine Reihe von Verbindungen des Molybdändichlorids mit Alkalihalogeniden beschrieben, denen er auf Grund seiner analytischen Resultate die allgemeine Formel $\mathrm{M}_{2}^{1} \mathrm{Mo}_{3} \mathrm{R}_{8}$. $2 \mathrm{H}_{2} \mathrm{O} \quad\left(\mathrm{M}^{\mathrm{1}}=\mathrm{K}, \mathrm{NH}_{4} ; \mathrm{R}=\mathrm{Cl}, \mathrm{Br}, \mathrm{J}\right)$ zuerteilt. Allerdings hat er nirgends vollständige Analysen, sondern überall nur einige Einzelbestimmungen ausgeführt.

Wurde eine konzentrierte Lösung der Verbindung $\mathrm{Mo}_{3} \mathrm{Cl}_{6} . \mathrm{HCl}$. $4 \mathrm{H}_{2} \mathrm{O}$ in Salzsäure 1.12 mit Ammoniak versetzt, so dafs die Lösung noch stark sauer blieb, so schied sich beim Stehen ein Gemisch feiner gelber Nadeln mit Ammoniumchlorid aus. Durch wiederholtes Unkrystallisieren aus Salzsäure wurde die Verbindung nach Möglichkeit von Chlorammonium getrennt. Die Analysenwerte, die mit vier verschiedenen Darstellungen erhalten wurden, liefsen die sichere Aufstellung einer Formel nicht zu.

\begin{tabular}{lrrrl}
\multicolumn{5}{c}{ Gefunden: } \\
$\mathrm{NH}_{4}$ & 5.60 & 5.51 & $5.11 \%$ & \\
$\mathrm{Mo}$ & 50.94 & 50.34 & 50.91 & $51.96 \%$ \\
$\mathrm{Cl}$ & 43.98 & 43.65 & 44.28 & 42.38 \\
$\mathrm{H}_{2} \mathrm{O}$ & Blomstrand findet $\mathrm{Cl}: 44.37 \%$ &
\end{tabular}




\begin{tabular}{lcccc} 
& \multicolumn{5}{c}{ Berechnet auf } \\
& $\left(\mathrm{NH}_{4}\right) \mathrm{Mo}_{3} \mathrm{Cl}_{7} . \mathrm{H}_{2} \mathrm{O}$ & $\left(\mathrm{NH}_{4}\right) \mathrm{Mo}_{3} \mathrm{Cl}_{7} . \mathrm{NH}_{3}$ & $\left(\mathrm{NH}_{4}\right) \mathrm{Mo}_{3} \mathrm{Cl}_{7}$ & $\left(\mathrm{NH}_{4}\right) \mathrm{Mo}_{3} \mathrm{Cl}_{8} .2 \mathrm{H}_{2} \mathrm{O}$ \\
BLOMSTRAND
\end{tabular}

Die gefundenen Werte stimmen am besten auf die Formel $\left(\mathrm{NH}_{4}\right) \mathrm{Mo}_{3} \mathrm{Cl}_{7}\left(\mathrm{NH}_{3}\right)$, die jedoch nach der Darstellung der Substanz aus saurer Lösung wenig wahrscheinlich erscheint; sie stimmen nicht auf die nach der Bildungsweise wahrscheinlichsten Formeln $\left(\mathrm{NH}_{4}\right) \mathrm{Mo}_{3} \mathrm{Cl}_{7} \cdot \mathrm{H}_{2} \mathrm{O}$ oder $\left(\mathrm{NH}_{4}\right) \mathrm{Mo}_{3} \mathrm{Cl}_{7}$, da der Ammoniakgehalt stets beinahe doppelt $\mathrm{zu}$ hoch gefunden wurde.

Auf die Formel BLomstrands endlich $\left(\mathrm{NH}_{4}\right)_{2} \mathrm{Mo}_{3} \mathrm{Cl}_{8} \cdot 2 \mathrm{H}_{2} \mathrm{O}$ stimmen die gefundenen Werte für Molybdän ganz und gar nicht. Diese wurden gewonnen, indem Molybdänsulfid zur Abscheidung gebracht und als Molybdäntrioxyd gewogen wurde und in anderen Fällen, indem die Verbindung im Wasserstoffstrome geglüht und das verbleibende metallische Molybdän gewogen wurde. Die nach diesen beiden Metboden erhaltenen Werte stimmen befriedigend überein. Die Frage nach der Zusammensetzung dieses Stoffes muls daher vertagt werden bis noch andere analoge Verbindungen mit anorganischen Halogeniden untersucht sind.

Wurde eine Lösung von $\mathrm{Mo}_{3} \mathrm{Cl}_{6} \cdot \mathrm{HCl} \cdot 4 \mathrm{H}_{2} \mathrm{O}$ in alkoholische Chlorwasserstoffsäure mit einer alkoholischen Lösung von Pyridiniumchlorid versetzt, so krystallisierte in tiefgelben Nadeln ein reines Pyridiniumdoppelsalz in fast quantitativer Ausbeute aus. Die Analyse dieser mehrfach dargestellten Verbindung führte zu der Formel $\left(\mathrm{C}_{5} \mathrm{H}_{5} \mathrm{~N}\right) \mathrm{H} \mathrm{MoCl}_{3} . \mathrm{H}_{2} \mathrm{O}$ oder wenn man dieselbe entsprechend den übrigen Verbindungen des „Dichlorids“ verdreifacbt:

$$
\left(\mathrm{C}_{5} \mathrm{H}_{5} \mathrm{~N}\right)_{3} \mathrm{H}_{3} \mathrm{Mo}_{3} \mathrm{Cl}_{3} \cdot 3 \mathrm{H}_{2} \mathrm{O} \text {. }
$$

Gefunden:

\begin{tabular}{lrrrrrr}
$\mathbf{N}$ & 4.66 & 5.07 & 5.15 & 4.68 & \multicolumn{2}{c}{$4.30 \%$} \\
Mo & 32.03 & 32.19 & 32.08 & 31.65 & 31.66 & $32.31 \%$ \\
Cl & 35.45 & 35.15 & 36.39 & $35.56 \%$ &
\end{tabular}

Berlin N, Wissenschaftlich-chemisches Laboratorium, 26. November 1909.

Bei der Redaktion eingegangen am 27. November 1909. 